\title{
EVALUACIÓN DE SOFTWARE LIBRE PARA LA GESTIÓN DE ARCHIVOS ADMINISTRATIVOS
}

\author{
José-Antonio Moreiro, Sonia Sánchez-Cuadrado, Vicente Palacios y \\ Eduardo Barra
}

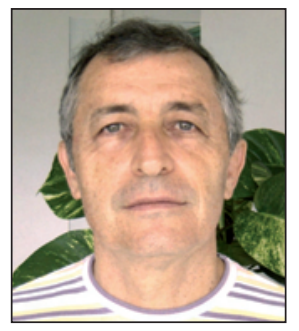

José-Antonio Moreiro es catedrático de biblioteconomía y documentación de la Universidad Carlos III de Madrid. Autor de trabajos sobre técnicas de análisis de contenido documental y cuestiones conceptuales de la documentación, ha publicado monografías, manuales y más de 60 artículos en revistas nacionales e internacionales. Ha participado en dos proyectos europeos y dirigido o colaborado en siete nacionales. Ha sido director del Departamento de Biblioteconomía y Documentación y decano de la Facultad de Humanidades, Comunicación y Documentación de la Universidad Carlos III de Madrid.

Departamento de Biblioteconomía y Documentación Universidad Carlos III de Madrid C/Madrid, 125. 28903 Getafe, Madrid joseantonio.moreiro@uc3m.es

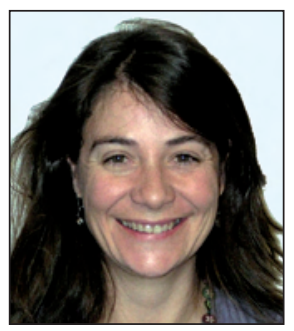

Sonia Sánchez-Cuadrado, doctora en documentación por la Universidad Carlos III de Madrid, desde 2002 es profesora en el Departamento de Informática de dicha universidad con docencia en las titulaciones de biblioteconomía y documentación e ingeniería informática. Sus principales áreas de investigación son la ingeniería de la información, la elaboración de sistemas de organización del conocimiento, la indización automática con procesamiento del lenguaje natural y la recuperación de información. Ha participado en 15 proyectos de investigación nacionales e internacionales.

Departamento de Informática Universidad Carlos III de Madrid 28911 Leganés, Madrid ssanchec@ie.inf.uc3m.es

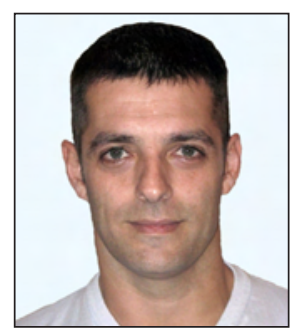

Vicente Palacios es ingeniero técnico en informática de gestión, ingeniero en informática y doctor en documentación por la Universidad Carlos III de Madrid. Imparte ingeniería del software desde el año 2000. Trabaja como coordinador de la Oficina de Software Libre de la UC3M donde se elaboran y evalúan nuevos proyectos y soluciones de software. Sus principales áreas de investigación son la web semántica, sistemas de gestión del conocimiento, ontologías y recuperación semántica. También investiga en áreas como software libre, ingeniería del software y desarrollo en java.

Departamento de Informática Universidad Carlos III de Madrid 28911 Leganés, Madrid palacios@di.uc3m.es

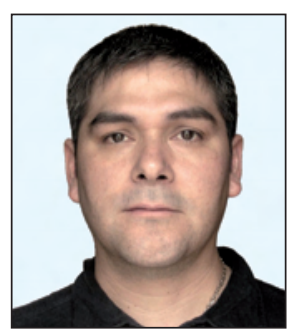

Eduardo Barra es graduado en ingeniería mecánico-eléctrica por la Universidad Veracruzana (México) e ingeniero en España. Desde el año 2004 es profesor de asignaturas relacionadas con la ingeniería del software en el Departamento de Informática en la Universidad Carlos III de Madrid. Entre sus principales áreas de investigación se encuentra la ingeniería de software y el paradigma de evolución de las tecnologías de la información. Ha participado en los principales congresos internacionales sobre estas materias.

Departamento de Informática Universidad Carlos III de Madrid 28911 Leganés, Madrid ebarra@ie.inf.uc3m.es

\section{Resumen}

Se evalúan tres aplicaciones de software libre de gestión de archivos administrativos para su implantación en el archivo de una universidad: Archon, Archivists' toolkit e ICA-AtoM. En la comparativa se tuvieron en cuenta doce requisitos concernientes a la gestión de metadatos, normas de descripción y préstamo, análisis y ciclo de vida del documento. Los resultados indican si el software posee determinadas características. Las aplicaciones analizadas no cumplen con todos los requisitos de un gestor de archivos, no obstante como software libre permiten la actualización e integración a nuevos contextos. 


\title{
Palabras clave
}

Software libre, Software de fuentes abiertas, Software libre, Gestor de archivo administrativo, Intranets.

\section{Title: Free software evaluation for administrative archives management}

\begin{abstract}
We evaluated three free software applications for administrative archives management and their implementation in university archives. Software evaluated was: Archon, Archivists' toolkit and ICA-AtoM. The comparative analysis considered 12 requirements concerning metadata management, description standards and lending, and document life-cycle analysis. The results show the requirements met by each software application evaluated. Although currently they do not satisfy all archive management requirements, open software can be customized and integrated with new contexts.
\end{abstract}

\section{Keywords}

Free software, Open source software, Administrative archive management, Intranets.

Moreiro, José-Antonio; Sánchez-Cuadrado, Sonia; Palacios, Vicente; Barra, Eduardo. “Evaluación de software libre para la gestión de archivos administrativos". El profesional de la información, 2011, marzo-abril, v. 20, n. 2, pp. $206-213$.

\section{Introducción}

Las instituciones suelen disponer de una intranet para el intercambio y la gestión de información administrativa, pero el tiempo que se requiere para buscar y obtener la que parezca más adecuada a veces es importante. Por ello pensamos que es útil la evaluación comparativa que presentamos en este artículo. Existen diferentes aplicaciones en el mercado, algunas genéricas y otras diseñadas para cada institución con archivos administrativos concretos o adaptados a la legislación de cada país, lo cual acarrea un coste económico. Katherine Wisser (2005) realizó un estudio sobre las aplicaciones para codificar y publicar documentos de acuerdo con EAD (encoded archival description). Los resultados concluyeron que según el tipo de organización existían dos clases: las grandes instituciones solían disponer de aplicaciones desarrolladas para sus propias necesidades, mientras que las pequeñas solían utilizar el software EAD Cookbook (Fox, 2002).

Por otra parte, pequeñas y grandes organizaciones consideran usar aplicaciones de software libre como una supuesta alternativa de menor coste. Existe por tanto la necesidad de evaluar su capacidad de configuración y adaptación a nuevos contextos, su coste a largo plazo y la existencia de comunidades de programadores y usuarios que ayuden en su mantenimiento y actualización.

Las organizaciones consideran el uso de software libre como una alternativa supuestamente de menor coste

En 2008, el Archivo de la Universidad Carlos III de Madrid (UC3M) solicitó a la Oficina de Software Libre (OSL) de dicha universidad una evaluación de las alternativas existentes de software libre para la gestión del archivo administrativo localizado en la intranet de la universidad. El estudio se realizó según los principios de evaluación de la funcionalidad y los requisitos particulares para el contexto concreto definido por los expertos del Archivo de la UC3M (OSL, 2009).

En este artículo se describe el proceso de evaluación realizado, comenzando con el análisis de estudios similares al propuesto por la OSL. A continuación se definen los requisitos necesarios para un gestor de archivos administrativos. Por último se analiza el cumplimiento de cada aplicación de los requisitos evaluados.

\section{Estudios relacionados}

Un sistema de gestión de archivos es un software que proporciona un soporte integrado para la vida de los documentos que incluye aspectos de elaboración, adquisición, evaluación, catalogación, almacenamiento, preservación, búsqueda y acceso.

Entre los sistemas de gestión de archivos libres están Archon, Archivists' toolkit (AT) e International Council on Archives-Access to Memory (ICA-AtoM).

La necesidad de definir criterios para evaluar el software de archivos administrativos ha generado diversos trabajos (Stevens, 2008; Spiro, 2009). Factores como el tamaño de la organización, la existencia de técnicos informáticos especializados en la plataforma, la necesidad de interoperabilidad y de publicación en web, entre otros, tienen un gran impacto en la selección de la solución idónea de un gestor de archivos administrativos. Westbrook et al. (2009) definieron algunos requisitos de alto nivel que se deberían cumplir para una integración entre $A T$ y Archon, centrándose en funciones de archivo, importaciones, exportaciones, registros, hojas de estilo, funciones de aplicación y acceso web. El informe de Spiro (2009) compara aspectos e informes de experiencias de archiveros en la implementación de sistemas de gestión de archivos. De las entrevistas con los expertos se determinaron 9 aspectos importantes para un software de este tipo:

1) integración con estándares para mejorar la interoperabilidad, y que permita búsquedas mediante $E A D$; 
2) permitir la importación sin tener que reprocesar los registros;

3) facilidad de exportación;

4) existencia de componentes para publicación web;

5) facilidad de uso, pero sin merma de las funciones necesarias para gestionar el ciclo de vida de los documentos;

6) rigurosamente basado en estándares como $E A D, D A C S$ (Describing archives: a content standard), o EAC (Encoded archival context), los cuales mejoran la corrección de los datos;

7) facilidad para gestionar la colección, con estadísticas, información sobre ubicaciones, posibilidad de registros temporales, etc.;

8) portabilidad, capacidad para trabajar offline y facilidad en la transferencia de los datos;

9) capacidad de planificar el trabajo asignando prioridades y mostrando indicadores para identificarlas.

En la tabla 1 se muestra una síntesis de algunas comparativas de software de gestión de archivos administrativos.

En el análisis de Spiro se destacan los siguientes factores: software libre; soporte (demo, formación, etc.); backups; formatos de importación/exportación: MARC, EAD, Dublin Core, MODS (metadata object description schema), METS (metadata encoding and transmission standard), MADS (metadata authority description schema), CSV (commaseparated values)...; gestión de la colección, ubicaciones, préstamos y prioridades, restricciones y derechos; gestión de informes y estadísticas; descripción mediante vocabularios controlados a diferentes niveles; jerarquías de registros; posibilidad de sindicación y recopilación automática; informes de errores; interoperabilidad; publicación en web; búsqueda y navegación web; posibilidad de enlazar imágenes; y facilidad de uso.
Hay otras aplicaciones libres que raramente figuran en las comparativas. Los motivos varían de un estudio a otro, pero básicamente son: el pequeño tamaño de la comunidad de usuarios, la inexistencia de soporte, la inmadurez de los proyectos o diseño inicial no dirigido a archivos. Algunos ejemplos de software no evaluados en el informe de Stevens (2008) son Hera2 (Quinn, 2000), Vernon CMS, OpenCollection (OSS) y Tabularium (Roberts, 2008), y fueron rechazados por su tangencial adaptación a los archivos y estar orientados principalmente a museos. En el caso concreto de ICA-AtoM fue rechazado para el informe de Stevens por inmadurez en el momento del estudio. Tabularium se desestimó por ser software gratuito pero no libre, y por tener escaso soporte y poca implantación entre los archiveros. Algunos de estos softwares son bases de datos adaptadas a archivos administrativos, como es el caso de Tabularium con Microsoft Access. Otro ejemplo es Archivematica, que integra un gran número de herramientas libres y se adapta a diversos estándares (como el modelo ISO-OAIS -open archival information system-). Esta aplicación está poco madura para ser considerada en las evaluaciones.

Un sistema de gestión de archivos es un software que proporciona un soporte integrado para la vida de los documentos

\section{Requisitos de un gestor de archivos administrativos para una universidad}

La Oficina de Software Libre (OSL) de la Universidad Carlos III de Madrid inició una búsqueda de software libre para la gestión de archivos administrativos que pudiera ofrecer una alternativa con las mismas funciones que el software propietario utilizado hasta entonces.

\begin{tabular}{|c|c|c|}
\hline Autor & Aplicaciones evaluadas & Foco \\
\hline Matienzo, 2006 & $\begin{array}{l}\text { Archivists' toolkit } \\
\text { Archon } \\
\text { ICA-AtoM }\end{array}$ & $\begin{array}{l}\text { Uno de los primeros análisis. Es un estudio somero. Enfatiza aspectos como madurez del producto, } \\
\text { facilidad de instalación, adecuación a la Web e implementación de normas de archivos. Destaca } \\
\text { el diseño adecuado para la gestión de archivos en ICA-AtoM, la financiación de AT y la usabilidad } \\
\text { de Archon. }\end{array}$ \\
\hline $\begin{array}{l}\text { Archivists' } \\
\text { toolkit, } 2008\end{array}$ & $\begin{array}{l}\text { Archivists' toolkit } 1.1 \\
\text { Archon } 2.1 \\
\text { PastPerfect } 4.0 \text { (no libre) }\end{array}$ & $\begin{array}{l}\text { Analiza un centenar de criterios sobre requisitos de instalación, gestión de datos, gestión de la } \\
\text { colección, formatos de importación y exportación, soporte para metadatos } E A D \text {, informes, perso- } \\
\text { nalización, y soporte de usuario. }\end{array}$ \\
\hline Stevens, 2008 & $\begin{array}{l}\text { Software libre: } \\
\text { Archivists' toolkit 1.1, } \\
\text { Archon } 2.2 \\
\text { Software propietario: } \\
\text { Archives Online } 3 \\
\text { PastPerfect } 4.0 \\
\text { Re:discovery Proficio } 8.6 \\
\text { STAR/Archives } 4.0, \\
\text { Filemaker Pro }\end{array}$ & $\begin{array}{l}\text { Incluye precio base, fortalezas y debilidades, y analiza } 6 \text { criterios: } 1 \text { ) desarrollo para gestión de } \\
\text { archivos, 2) existencia de soporte en Nueva Escocia, 3) capacidad de acceso y descripción de regis- } \\
\text { tros, 4) organización de registros jerárquicamente, 5) comunidad de usuarios amplia, 6) centrado } \\
\text { en el mercado inglés. } \\
\text { Los más destacados fueron STAR, AT y Archives Online. También se realizaron entrevistas a archive- } \\
\text { ros sobre su experiencia en usabilidad de las aplicaciones. }\end{array}$ \\
\hline Spiro, 2009 & $\begin{array}{l}\text { Software libre: } \\
\text { Archon } \\
\text { Archivists' toolkit } \\
\text { CollectiveAccess } \\
\text { Software propietario: } \\
\text { Cuadra STAR, } \\
\text { Eloquent } \\
\text { Minisis M2A }\end{array}$ & $\begin{array}{l}\text { Se trata del trabajo más extenso que se ha llevado a cabo hasta la fecha. Su objetivo es mejorar el } \\
\text { proceso de colecciones escondidas (hidden collections), es decir archivos con escaso control perte- } \\
\text { necientes a colecciones especiales. } \\
\text { El método de trabajo se basó en entrevistas, revisión bibliográfica y análisis de sitios web. El aná- } \\
\text { lisis contempla múltiples criterios, destacando: descripción de las colecciones, adquisición (libre } \\
\text { o propietario), costes, posibilidad de hosting, mantenimiento a largo plazo (p. e. si desaparece el } \\
\text { creador), calidad del soporte a usuarios, madurez y fiabilidad de la herramienta, existencia de infor- } \\
\text { mes y estadísticas, capacidad de configurar la gestión, capacidad de enlazar objetos y publicación } \\
\text { y exportación a la Web, posibilidad de trabajar sin conexión, y soporte a estándares de archivos. }\end{array}$ \\
\hline
\end{tabular}

Tabla 1. Estudios de sistemas de gestión de archivos 
Primero se consultó a los expertos del Archivo de la UC3M cuáles eran los requisitos que debía cumplir, como por ejemplo:

- los datos deben ser introducidos y almacenados de acuerdo con las normas internacionales $I S A D(G)$, mediante la configuración de plantillas hechas a medida para los diferentes grupos de usuarios;

- la descripción del documento debe poder incluir el objeto digital (imagen, .pdf, .doc, etc.)

- permitir la consulta mediante criterios diversos y de forma sencilla y avanzada;

- posibilitar el almacenamiento y consulta de los metadatos de cada registro con la información del usuario que lo ha creado, la fecha, y quién lo ha modificado;

- permitir la gestión de usuarios con diversos derechos de acceso;

- cada documento debe tener establecidas unas condiciones de seguridad y accesibilidad para su consulta.

Se elaboró un conjunto de 31 requisitos que en este artículo se han resumido a 12 fundamentales en relación con la gestión de metadatos, las normas de descripción y préstamo, y el análisis y ciclo de vida del documento (tabla 2).

\section{Análisis de los sistemas libres de gestión de archivos}

\section{Archon}

Elaborado por la University of Illinois, incluye la automatización de algunas tareas y la simplificación de codificaciones en formatos como MARC y EAD. Sus autores lo definen como un software de fácil uso. Está traducido al español, al gallego y al portugués y está pendiente la traducción al catalán y al euskera.

Las principales características técnicas orientadas al usuario final son la capacidad de búsqueda de materiales electró- nicos, la visualización, la impresión y la navegación. Los encargados de almacenar el contenido de la colección disponen de funciones tales como crear descripciones estándar; describir las series, subseries, archivos, ítem, etc.; editar; exportar, etc.

\section{El gestor de archivos administrativos debe} posibilitar el almacenamiento y consulta de los metadatos de cada registro

\section{Características técnicas (Archon, 2009):}

Los usuarios finales pueden:

- realizar búsquedas simultáneas de descripciones, registros y otros objetos digitales;

- disponer de modos de visualización, descarga, impresión y ayuda para búsqueda en colecciones individuales;

- navegar de forma sencilla entre objetos digitales y descripciones de archivos;

- visualizar por título de la colección o del objeto digital, encabezamiento de materia, autor, o grupos de archivos.

\section{Los usuarios encargados de gestionar el archivo pueden:}

- crear descripciones estándar, utilizando formularios web de ayuda;

- describir series, subseries, ficheros, etc., para cada colección;

- organizar colecciones dentro de un grupo o subgrupo de registro según su finalidad u origen;

- elaborar listas de autoridades y materias, enlazándolas con las colecciones y los ficheros;

- registrar la ubicación para buscar y encontrar nuevos materiales;

- subir ficheros y describir url externas;

\begin{tabular}{|c|c|c|}
\hline Código & Denominación & Descripción \\
\hline R1 & Descripción archivística & $\begin{array}{l}\text { Introducción de datos conforme a normas internacionales } I S A D(G) \text {. Configuración de planti- } \\
\text { llas a medida para distintos grupos de usuarios }\end{array}$ \\
\hline R2 & Consulta & Búsquedas (sencilla/avanzada) en bases de datos con criterios diversos \\
\hline R3 & Préstamo & Gestión y control de préstamo y devolución de documentos al archivo general \\
\hline R4 & Impresión & $\begin{array}{l}\text { Generación de etiquetas impresas e informes con listados de las diversas consultas que pue- } \\
\text { dan realizarse en la aplicación, con formato configurable a necesidades diferentes }\end{array}$ \\
\hline R5 & Gestión de usuarios & $\begin{array}{l}\text { Definición de grupos de usuarios con diferentes derechos de acceso tanto a funciones como } \\
\text { a información }\end{array}$ \\
\hline R6 & Transferencias & $\begin{array}{l}\text { Control de la situación de la documentación dentro de su ciclo de vida: archivos de oficina/ar- } \\
\text { chivo general en relación con el calendario de transferencias }\end{array}$ \\
\hline R7 & Gestión de espacios & $\begin{array}{l}\text { Ubicación de la documentación en los depósitos, y gestión lo más visual posible desde la } \\
\text { aplicación. Optimización de huecos }\end{array}$ \\
\hline R8 & Explotación estadística & $\begin{array}{l}\text { Generación de estadísticas de préstamo, transferencia, volumen de documentación gestio- } \\
\text { nada, etc. }\end{array}$ \\
\hline R9 & $\begin{array}{l}\text { Gestión de normas de conservación / } \\
\text { series documentales }\end{array}$ & $\begin{array}{l}\text { Cada documento tiene establecidas unas condiciones de accesibilidad (plazo y personas au- } \\
\text { torizadas a su consulta y préstamo) además de un plazo de conservación, transcurrido el cual } \\
\text { debe ser destruido. El programa debe facilitar la gestión de ambos aspectos }\end{array}$ \\
\hline R10 & Gestión de documentos electrónicos & $\begin{array}{l}\text { La descripción de un documento (metadatos) debe poder incluir el objeto digital (imagen, } \\
\text {.pdf, .doc, etc.) }\end{array}$ \\
\hline R11 & $\begin{array}{l}\text { Almacenamiento y consulta de los } \\
\text { metadatos de cada registro }\end{array}$ & $\begin{array}{l}\text { Posibilidad de almacenar y consultar los metadatos de cada registro, qué usuario lo ha crea- } \\
\text { do, en qué fecha, quién lo ha modificado, etc. }\end{array}$ \\
\hline R12 & Documentación & Calidad de la documentación de la plataforma \\
\hline
\end{tabular}

Tabla 2. Definición de los requisitos de un gestor de archivos administrativos 
- editar información descriptiva desde una interfaz pública;

- exportar a formatos MARC y EAD;

- listar materiales sin procesar.

Los usuarios administradores de $\mathrm{Ar}$ chon pueden:

- añadir direcciones de contacto;

- definir grupos de registros y otras clasificaciones;

- configurar la interfaz pública por temas;

- crear vistas de los datos;

- añadir, editar o borrar cuentas de usuarios, y validar el acceso;

- importar archivos con formatos $M A R C, E A D(\mathrm{xml})$ o CSV.

El número de instituciones que ha implantado el software Archon es reducido. Destacan archivos históricos, organizaciones sin fines de lucro y entidades educativas como la University of San Diego, la University of Illinois (figura 1) y la University of Massachusetts. En España lo tiene el Ateneu Barcelonès (Montes; VicenteHernández, 2009).

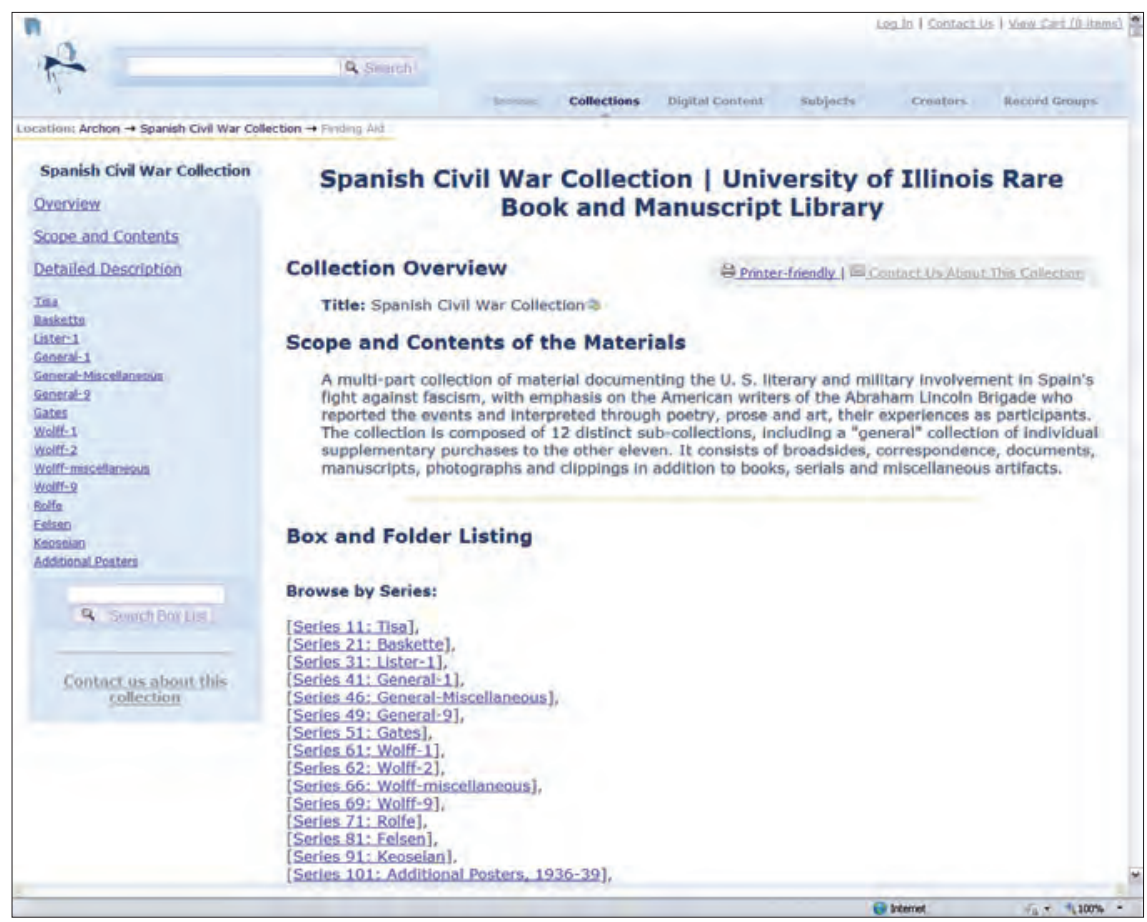

Figura 1. Software Archon para la colección de libros raros de la University of Illinois

AT permite la descripción y exportación de materiales de archivo en los formatos $E A D$ y $M A R C$

\section{Archivists' toolkit}

El consorcio formado por las bibliotecas de la University of California-San Diego y Nueva York junto con otras instituciones han elaborado el software libre para la gestión de archivos Archivists' toolkit (AT) mantenido por The Andrew W. Mellon Foundation. AT trata de reducir los costes de formación del personal. Permite la descripción y exportación de materiales de archivo en los formatos $E A D$ y $M A R C$, y el control de autoridades y la codificación descriptiva según el estándar para objetos administrativos METS.

\section{Características técnicas:}

- soporte integrado para gestión de materiales de archivo procedentes de la adquisición de repositorios, identificación de recursos o donaciones, adquisición de registros, control de autoridades, descripción de recursos de archivos y objetos digitales, gestión para la localización de información;

- interfaz adaptable;

- modificación de niveles de campos;

- orientación con valores por defecto para campos y notas que pueden ser reutilizadas de forma común o estandarizada como condiciones de uso;

- adaptación de los campos de búsqueda;

- inserción de datos legales en formatos como EAD 2002 o MARC xml;
- fácil incorporación de datos para crear listados;

- gestión de cuentas de usuarios con niveles de permisos y control de acceso;

- exportación de datos a formatos como EAD 2002, MARC xml, METS, MODS y Dublin Core;

- posibilidad de trabajo en local o dentro de una red con múltiples repositorios.

http://archiviststoolkit.com

La Historical Society of Pennsylvania es una entidad relevante en la conservación de archivos en Estados Unidos. Cuenta con el proyecto "Fondly, Pennsylvania: notes from archives and conservation" que implementa el software AT con el fin de estandarizar la administración de documentos y favorecer las migraciones futuras a cualquier otro sistema (figura 2).

\section{ArchivesSpaces}

La iniciativa denominada ArchivesSpaces project tiene como objetivo la fusión de Archon y AT. Está en fase de definición de requisitos y de evaluación de las distintas opciones de implementación (Matienzo, 2010). Se espera que el resultado pueda suplir las carencias de Archon en relación con la funcionalidad web. El resultado previsto será una arquitectura SOA (orientada al servicio), que permite que los componentes sean accesibles de forma independiente. Se ha planificado una arquitectura de tipo REST (representational state transfer), esto es, una arquitectura que mejore la integración con la web. Además de estos requisitos básicos Matienzo sugiere distintas posibilidades en relación con el lenguaje de programación, la persistencia y el almacenamiento, la importación y exportación de datos, la indización y la búsqueda, así como cuestiones relativas a la interfaz de usuario, autenticación, workflow, informes y licencias.

Las alternativas de software libre tienen evidentes ventajas, pero también hay que considerar otros factores. En 2006 Matienzo ya mostró la preocupación por el mantenimiento de nuevos proyectos que no contaran con una comunidad de 
usuarios. Sibyl Schaefer (2010), analista de $A T$ entre 2007 y 2009 , menciona algunos problemas que tiene la plataforma. La comunidad de usuarios debe ser lo suficientemente grande para hacer sostenible el proyecto y se les debe delegar el mantenimiento, especialmente después de la fusión entre $A T$ y Archon. Es necesario hacer más fácil la instalación y mejorar la usabilidad y el mantenimiento del software. También hace falta implementar una estructura que permita a los usuarios colaborar eficientemente con el proyecto (por ejemplo coordinación, documentación y formación adecuada).

\section{ICA-AtoM (International Council on Archives-Access to Memory)}

Es una aplicación en código abierto para la descripción archivística llevada a cabo por Peter Van Garderen, presidente la consultora ArteFactual Systems en colaboración con la National School for Archivists (Rijksarchief School) de Holanda.

\section{http://www.artefactual.com}

Esta iniciativa se enmarca dentro de un programa del International Council on Archives (ICA) con financiación de la Unesco. Desde el grupo de trabajo "Archivos y derechos humanos", se ha creado una guía online de fuentes de archivo para el estudio de las violaciones de los derechos humanos. Las descripciones se realizan de acuerdo con las normas ISAD (G), Isaar (CPF), e ICA-Isiah, y los puntos de acceso de materia se basan en el tesauro de la Unesco. La interfaz ofrece acceso mediante búsqueda sencilla por palabras y navegación por materia, idioma, país, centro de archivo y fecha.

En noviembre de 2010 presentaron su primera versión no beta (v. 1.1) incorporando nuevas características como la importación y exportación de documentos SKOS y EAC-CPF (figura 3).

\section{Características técnicas de la v. 1.1:}

- comprobación de actualizaciones;

- nueva interfaz de búsqueda;

- incorporación de plugin de metadatos para $D C, I S A D, R A D$, Isaar.

- crear, editar y eliminar las funciones de los registros de autoridad.

- cumplimiento de las normas siguientes:

$I S A D(G)=$ General international standard archival description. 2a edición, 1999.

Isaar $(C P F)=$ International standard archival authority record -for corporate bodies, persons and families-, 2a ed., 2003.

Isdiah = International standard for describing institutions with archival holdings, 1a ed., marzo de 2008.

- búsqueda avanzada;

- opciones de filtrado por las descripciones de archivo como el tema, el nombre, el lugar, el tipo de material, etc.;
- visualizar los archivos digitales creados de forma automática;

- interfaz de usuario multilingüe.

http://ica-atom.org/doc/Category:Releases

\section{Proceso de evaluación}

Ha consistido en valorar la cobertura que cada una de las alternativas para la gestión de archivos ofrece a los 12 requisitos de la tabla 2 .

De hecho, este trabajo continúa el realizado en 2009 por la Oficina de Software Libre de la UC3M y lo actualiza utilizando las últimas versiones liberadas de cada plataforma: Archon v. 3.14, Archivists' toolkit 2.0 update 10, e ICA-AtoM 1.1. Para cada una de ellas se ha consultado la documentación incluida en manuales de usuarios, foros, wikis, etc., completándose con los programas de demostración que proporcionan los candidatos. El resultado de la evaluación se muestra en la tabla 3.

Los tres sistemas satisfacen el primer requisito (R1), es decir, permiten la descripción de documentos conforme a normas internacionales y personalizar la entrada conforme a distintos perfiles de usuario. Este aspecto facilita la importación y exportación de registros, así como la descripción de documentos por los usuarios del sistema, pudiéndose simplificar

\begin{tabular}{|l|c|c|c|c|c|c|c|c|c|c|c|c|}
\hline & $\mathrm{R} 1$ & $\mathrm{R} 2$ & $\mathrm{R} 3$ & $\mathrm{R} 4$ & $\mathrm{R} 5$ & $\mathrm{R} 6$ & $\mathrm{R} 7$ & $\mathrm{R} 8$ & $\mathrm{R} 9$ & $\mathrm{R} 10$ & $\mathrm{R} 11$ & $\mathrm{R} 12$ \\
\hline Archon & $\mathrm{x}$ & $\mathrm{x}$ & & & $\mathrm{x}$ & $\mathrm{x}$ & $\mathrm{x}$ & & $\mathrm{x}$ & $\mathrm{x}$ & $\mathrm{x}$ & $\mathrm{x}$ \\
\hline $\begin{array}{l}\text { Archivists' } \\
\text { toolkit }\end{array}$ & $\mathrm{x}$ & $\mathrm{x}$ & & $\mathrm{x}$ & $\mathrm{x}$ & $\mathrm{x}$ & $\mathrm{x}$ & $\mathrm{x}$ & $\mathrm{x}$ & $\mathrm{x}$ & $\mathrm{x}$ & $\mathrm{x}$ \\
\hline ICA-AtoM & $\mathrm{x}$ & $\mathrm{x}$ & & & $\mathrm{x}$ & $\mathrm{x}$ & $\mathrm{x}$ & $\mathrm{x}$ & & $\mathrm{x}$ & $\mathrm{x}$ & $\mathrm{x}$ \\
\hline
\end{tabular}

Tabla 3: Resultados de la evaluación de los gestores de archivos administrativos de software libre 


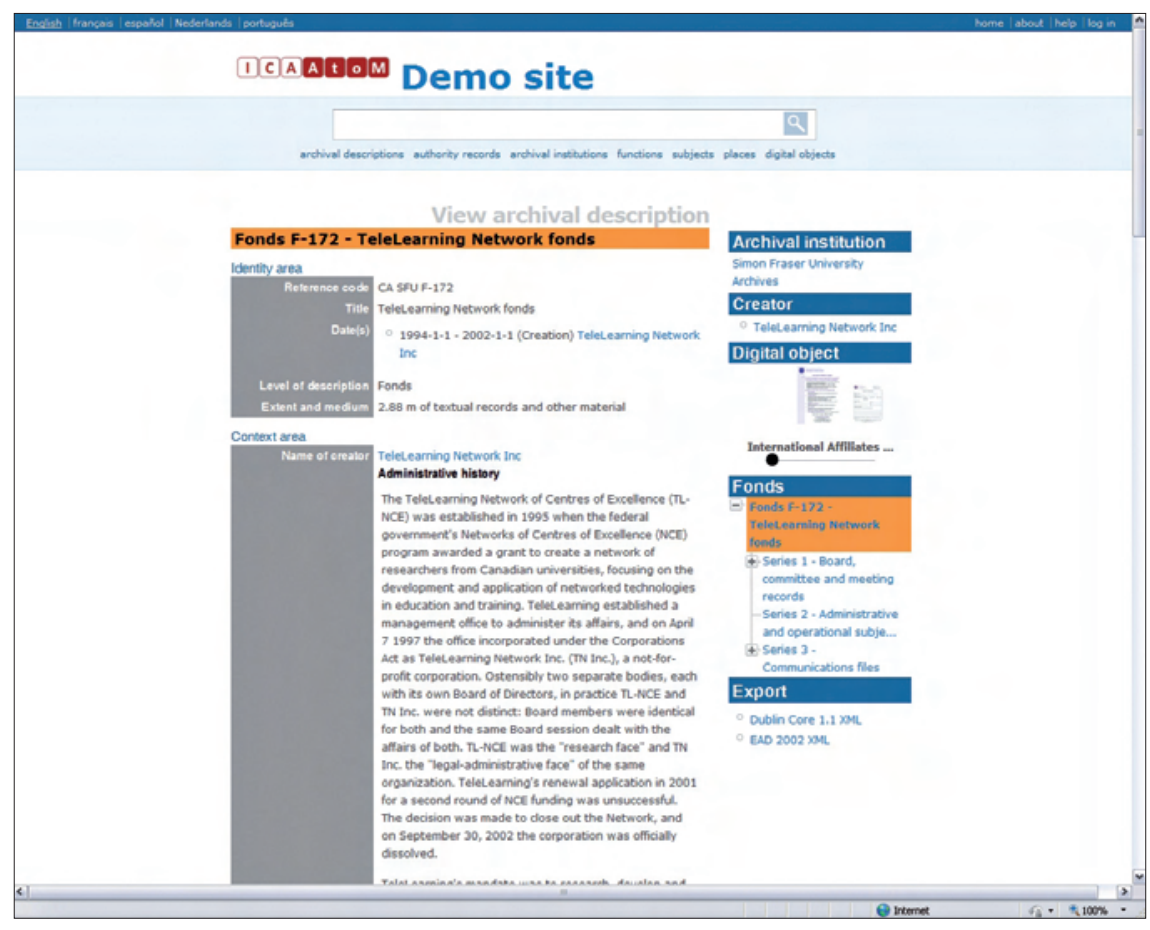

Figura 3. Versión demo de ICA-AtoM

y personalizar las pantallas de introducción de datos en función del rol de cada usuario.

El segundo aspecto (R2) también es cubierto por los tres, dado que todos ellos permiten realizar búsquedas sencillas y avanzadas con distintos criterios. Esto facilita la recuperación de los documentos.

En la tercera característica (R3) se evalúa la gestión del préstamo y devolución de documentos. Ninguno de los candidatos lo cubre.

R4 es la posibilidad de generar etiquetas e informes impresos con formato configurable. Sólo $A T$ soporta este requisito, si bien está planificado para futuras versiones de ICAAtoM. Archon proporciona plantillas para su personalización por parte de programadores.

La definición de usuarios, grupos y derechos de acceso, tanto a la funcionalidad de la aplicación como a la información gestionada por el sistema (R5) la cumplen las tres aplicaciones, siendo, de menor a mayor funcionalidad: Archon y $A T$, basados en roles e ICA-AtoM que incluye roles y $A C L S$ (access control lists).

La sexta (R6) se corresponde con la gestión de la documentación dentro de su ciclo de vida, desde su generación a su transferencia al archivo general desde los archivos de oficina. Este aspecto es cubierto por los tres softwares, aunque es $A T$ el que proporciona la cobertura más completa, limitándose en los otros dos al establecimiento de ubicaciones y prioridades.

R7, gestión de depósitos del modo más usable posible e incluyendo ayudas a la optimización, lo presentan los tres candidatos. De menor a mayor capacidad de descripción se encuentran: ICA-AtoM, que almacena básicamente etiquetas; Archon, que permite realizar una descripción detallada del tamaño de los documentos, así como su ubicación física; y $A T$, que soporta una descripción pormenorizada, lo que facilita la optimización del espacio. No obstante, no se realiza de forma visual y deja a cargo del administrador la distribución del espacio físico.

La octava característica (R8), estadísticas del proceso y uso de la documentación, sólo es soportada por $A T$, con un módulo específico, si bien ICA-AtoM tiene planificado un desarrollo similar.

La gestión de políticas de acceso y conservación de los documentos (R9) la brindan tanto Archon como AT, que soportan la gestión de materiales restringidos. ICA-AtoM aún está pendiente de incluirla. Las políticas de conservación, relacionado con el sexto requisito, están mejor resueltas por $A T$, frente a los otros candidatos, que básicamente ofrecen anotaciones.

La décima característica (R10) es la posibilidad de incluir objetos digitales tales como imágenes, documentos, etc. Los tres softwares lo hacen incluyendo incluso, en el caso de ICA-AtoM, la reproducción de material multimedia.

La undécima (R11) se corresponde con la posibilidad de crear registros de metadatos, incluyendo información sobre su creación y modificación. Esto es soportado por los tres sistemas, de forma muy simple en el caso de $A T$, más elaborada en Archon y compatible con estándares internacionales en ICA-AtoM.

Duodécima característica (R12), calidad de la documentación de la plataforma: Archon posee una documentación poco detallada, pero actualizada. $A T$ tiene una documentación extensa y detallada, pero no actualizada, siendo la última versión disponible la 1.5.9. Por último, ICA-AtoM dispone de un wiki con información poco detallada, aunque actualizada.

Para finalizar la evaluación es preciso matizar algunos aspectos de las licencias:

Licencia Archon: permite usar, copiar, modificar, redistribuir, relicenciar y vender el producto (y derivados) siempre y cuando el código que se distribuya mantenga copyright, disclaimer (descargo de responsabilidad) y las mismas condiciones que el producto original. No se puede usar el nombre de los creadores de Archon para promocionar un producto derivado.

http://www.archon.org/license.php

Licencia Archivists' toolkit: similar a la de Archon con la salvedad de que se debe adjuntar una hoja de cambios en la que se indique fecha y modificaciones y/o aportaciones realizadas.

http://www.archiviststoolkit.org/downloads/license.shtml

Licencia ICA AtoM: bajo licencia GPLv2 (General public Iicense, $v$. 2), de forma que se pueden realizar todo tipo de 
cambios en el código fuente y redistribuir libremente nuevas versiones. Además se permite copiar, modificar y redistribuir, siempre bajo licencia GPLv2.

http://www.ica-atom.org/about.html

Las dos primeras licencias son similares a la BSD (Berkeley software distribution), mucho menos restrictiva que la GPLV2, la cual, con el fin de garantizar las libertades de los usuarios en las obras derivadas, obliga a que éstas sean distribuidas bajo la misma licencia. Este efecto viral, implícito en la licencia GPLV2, garantiza las libertades de los usuarios, pero puede dificultar el desarrollo de nueva funcionalidad a programadores reticentes a la imposición del licenciamiento GPLV2.

Una de las grandes ventajas que tiene el software libre para archivos es su mantenimiento y actualización

\section{Conclusiones}

Una de las grandes ventajas que tiene el software libre para archivos es su mantenimiento y actualización. El desarrollo digital de un archivo es una labor a largo plazo. Las empresas que crean software propietario están sujetas a los vaivenes de la industria. Una empresa rentable puede no ser viable pasado el tiempo y desaparecer, desapareciendo al tiempo el software al que daba soporte. Esto convierte al software libre en una alternativa prometedora en la gestión de archivos. La aparición casi continua de actualizaciones de estas aplicaciones apoya las anteriores afirmaciones. Si bien es cierto que ninguna aplicación de las evaluadas responde al cien por cien a los requisitos de una institución determinada, el software libre permite la actualización y la integración a nuevos contextos. El que sea factible integrar y adaptar no significa que no existan costes económicos. No obstante, una evaluación previa de las distintas alternativas puede reducirlos considerablemente.

\section{Bibliografía citada}

Archon. "Features matrix: Archivists' toolkit, Archon, and PastPerfect". Archivists' toolkit for archivists by archivists, 2009.

http://archiviststoolkit.org/node/76

CNSA. "Archives management software: template features table". Council of Nova Scotia Archives, 2008.

http://www.councilofnsarchives.ca/resources/SoftwareRe view.htm

Calvo-Vidal, Oskar. "Archon, software libre para gestionar archivos". http://www.soft-libre.net/docs/archon.pdf

CollectiveAcess. CollectiveAccess: web based software to catalogue, manage and publish museum and archival collections, 2011. http://www.collectiveaccess.org/download

Fox, Michael J. EAD Cookbook. 2002.

http://www.archivists.org/saagroups/ead/ead2002cookbo okhelp.html

ICA-Atom. ICA-Atom: open source archival description software, 2011.

http://ica-atom.org

Matienzo, Mark A. ArchivesSpace technical design meetig, 2010.

http://archivesspace.org/documents/ArchivesSpace_Tech nical_Architecture_Report-FINAL-07302010.pdf

Matienzo, Mark A. "The state of open source archival management software". Thesecretmirror.com, 2006.

http://thesecretmirror.com/blog/2006/12/21/the-state-ofopen-source-archival-management-software

Montes, Sergi; Vicente-Hernández, Lluís. "La memoria en abierto: la implantación del software Archon para la gestión del archivo digital del Ateneu Barcelonés". Fesabid'09, pp. 267-274.

http://www.fesabid.org/zaragoza2009/actas-fesabid2009/267-274.pdf

OSL UC3M. Evaluación de software libre para gestión de archivos. Oficina de software libre de la UC3M, 2009.

http://crisol.uc3m.es/index.php/top-noticias/37-alternati vas-libres/45-s/gestionarchivos

Quinn, Cliff. Hera2, 2000.

Roberts, David. The tabularium website, 2008.

http://tabularium.records.nsw.gov.au

Schaefer, Sibyl. "Challenges in sustainable open source: a case study". Journal code\{4\}lib, 2010, n. 9.

http://journal.code4lib.org/articles/2493

Schwartz, Scott W. et al. Archon: the simple archival information system, 2011.

http://www.archon.org

Spiro, Lisa. "Archival management software". CLIR Reports, 2009.

http://clir.org/pubs/reports/spiro2009.html

Stevens, Amanda. Archives management software review report. Council of Nova Scotia Archives, 2008.

http://www.councilofnsarchives.ca/resources/CNSASoftware Review 2008-Final.pdf

Universidad de Illinois. Archon. User manual. http://www.archon.org/UserManualv2.2.pdf

Vernon Systems. Collections management software for museums, 2010.

http://www.vernonsystems.com

Westbrook, Brad et al. Archivists' toolkit, 2009. http://archiviststoolkit.org

Wisser, Katherine M. "EAD tools survey", 2005. http://www.archivists.org/saagroups/ead/EADToolsSurvey. $p d f$ 